\title{
Mortalidad natural de larvas de merluza común (Merluccius gayi gayi) frente a Chile centro-sur en relación a su distribución espacial y grado de agregación*
}

\author{
Cristian Vargas ${ }^{1}$, Guillermo Valenzuela ${ }^{1}$, Sergio Núñez ${ }^{2}$ y Dagoberto Arcos ${ }^{2}$ \\ ${ }^{1}$ Instituto de Biología Marina "Dr. Jürgen Winter", Universidad Austral de Chile, Casilla 567, Valdivia, Chile \\ ${ }^{2}$ Instituto de Investigación Pesquera Octava Región S.A., Casilla 350, Talcahuano, Chile.
}

RESUMEN: Existe escasa información sobre la ecología y sobrevivencia de estados larvales de merluza común frente a las costas de la VIII Región, Chile. Debido a que la agregación puede ser un factor determinante en la depredación de larvas de peces, en el presente trabajo se estudia su influencia en la mortalidad natural instantánea de larvas de merluza común (Merluccius gayi gayi Guichenot, 1848), estimada a través del modelo mortalidad-agregación de McGurk (1986). Las muestras (red bongo 500 $\mu$ ) fueron obtenidas durante un crucero efectuado en la zona costera del centrosur de Chile (36 $\left.22^{\prime} \mathrm{S}-37^{\circ} 10^{\prime} \mathrm{S}\right)$ durante septiembre de 1991.

La tasa de mortalidad natural instantánea fluctuó entre 0,1 y $0,38 \mathrm{~d}^{-1}$ para larvas en un rango de tallas de 4 a 11 mm de longitud estándar. Los valores de mortalidad obtenidos a través del modelo de interacción mortalidad-agregación (McGurk, 1986), revelaron que la distribución espacial (agregación) podría influenciar considerablemente a la mortalidad, especialmente en larvas de mayor longitud (>9 $\mathrm{mm})$.

Palabras claves: mortalidad natural, Merluccius gayi gayi, distribución espacial, agregación.

\section{Natural mortality of chilean hake (Merluccius gayi gayi) larvae of central-south Chile in relation to their spatial distribution and patchiness"}

\begin{abstract}
At the present time limited information is available about ecology and survival of Chilean hake larvae off coast from VIII Region, Chile. Because spatial patchiness of larvae may be an important factor on the predation of fish larvae, its influence on the instantaneous natural mortality of larval Chilean hake (Merluccius gayi gayi Guichenot, 1848) was studied using the mortality-patchiness model (McGurk, 1986). Samples (bongo net 500 $\mu$ ) were taken during a survey carried out in the coastal zone off Central-South Chile (36 $\left.22^{\prime} \mathrm{S}-3^{\circ} 10^{\prime} \mathrm{S}\right)$ during september 1991.

The natural mortality for larvae between 4 to $11 \mathrm{~mm}$ of standard length fluctuated between 0,1 and $0,38 \mathrm{~d}^{-1}$. The mortality values obtained using the mortality-patchiness model (McGurk, 1986), showed that the spatial distribution (patchiness) would influence the larval mortality, specially in the largest ones (>9 mm).
\end{abstract}

Key words: natural mortality, Merluccius gayi gayi, spatial distribution, patchiness.

\section{INTRODUCCION}

La inquietud por identificar las causas de las fluctuaciones en la abundancia de una nueva clase anual de peces no es reciente. Al respecto, durante las últimas décadas se le ha conferido una real importancia a los estudios sobre mortalidad larval, los que parecieran ser la clave para determinar los factores que controlan el destino de las poblaciones de peces. Altas tasas de mortalidad son atribuidas gene- ralmente a la depredación o inanición de embriones y larvas (Hunter, 1976; 1981), siendo la depredación, más que la muerte fisiológica por inanición, la mayor causa de mortalidad larval (Wroblewski, 1984). Sin embargo, ambas fuentes pueden interactuar, debido a que un pobre estado nutricional puede hacer a las larvas más vulnerables a la depredación (Shepherd y Cushing, 1980).

Es sabido que huevos y larvas de peces presentan una distribución espacial agrupada o en "par-

\footnotetext{
* Proyecto DID - UACH: S-94-59.
} 
ches" (Smith, 1973; Haury et al., 1978; Houde y Lovdal, 1985). Esto involucra altas tasas de mortalidad natural, producto de la atracción de depredadores en función del tamaño o densidad de "parches" de huevos y/o larvas, o respuestas reproductivas de una población depredadora a la producción de estos parches (Hunter, 1976).

Varios han sido los modelos matemáticos que han tratado de explicar los procesos que influencian la tasa de mortalidad en organismos marinos. Algunos se han basado en los mecanismos físico-biológicos que afectarían a las larvas de peces (Wroblewski, 1984), mientras que otros en la depredación dependiente de la longitud (Ware, 1975; Peterson y Wroblewski, 1984). McGurk (1986) ha sido uno de los pocos autores en crear un modelo en el cual la depredación no ha sido asumida como constante, sino como un factor que depende de la distribución espacial tanto del depredador como de la presa.

McGurk (1986) agrega que este modelo tiene un significado biológico, basado en la idea de que huevos y larvas de peces son más vulnerables a los depredadores a medida que aumenta su nivel de agregación en el ambiente. Su vulnerabilidad implica que la tasa de alimentación de sus depredadores está limitada más por el tiempo requerido en localizarlas que por el tiempo en capturarlas y consumirlas; por lo tanto, la distribución espacial de huevos y larvas de peces determina cuan a menudo un depredador encuentra un parche de huevos o larvas y cuan intensa es la mortalidad por depredación dentro de éste. Finalmente, el autor sostiene que este modelo puede ser generalizado para cualquier interacción depredador-presa.

La zona costera frente a Chile centro-sur caracterizada por un significativo proceso de surgencia costera, ha desarrollado una importante pesquería pelágica y demersal (Arcos, 1992). Dentro de las principales especies demersales capturadas por la flota arrastrera, destaca la merluza común (Merluccius gayi gayi Guichenot, 1848). Dicha especie realiza sus procesos reproductivos en esta área (Vargas, 1995), presentando un período de desove principal entre los meses de septiembre y octubre (Furet y Lépez, 1984; Amín et al., 1989; Alarcón, 1992).

Las significativas concentraciones de ésta y otras especies en la zona costera frente a Talcahuano, parecieran estar asociadas a la alta productividad aportada por los procesos de afloramiento costero, características topográficas de la línea de costa y la compleja batimetría de la zona (i.e. terrazas y cañones) cuyo efecto sobre la dirección del flujo geostrófico, genera anillos anticilónicos que aumentan el tiempo de residencia de las aguas de surgencia (Sepúlveda, 1990; Cáceres y Arcos, 1991) y la retención de huevos y larvas de peces en áreas geográficas, relativamente limitadas (Vargas, 1995).

El objetivo central del presente trabajo es, desde la perspectiva de una mesoescala de observación, determinar el rol de la distribución espacial durante el período de desove de la especie, en la tasa de mortalidad natural instantánea diaria de estados larvales de Merluccius gayi gayi, estimada a través del modelo de interacción mortalidad-agregación de McGurk (1986).

\section{MATERIALES Y METODOS}

\section{Área de estudio}

El área de estudio (Fig. 1) comprende la zona nerítico-costera del centro-sur de Chile abarcando un rango latitudinal entre los $36^{\circ} 22^{\prime} \mathrm{S}$ y $37^{\circ} 10^{\prime} \mathrm{S}$. El sector está influenciado estacionalmente por un proceso de surgencia costera en el período comprendido entre los meses de septiembre y marzo (Arcos y Navarro, 1986; Arcos, 1987), cuando los vientos provienen principalmente del sur y suroeste (Wooster y Reid, 1963; Arcos y Navarro, 1986; Arcos, 1986).

La zona se caracteriza también (Fig. 1) por presentar un conjunto de bahías y golfos y las más ancha plataforma del país (terraza del Itata) limitada por los cañones submarinos de los ríos Itata y BíoBío.

\section{Diseño del muestreo}

Las muestras ictioplanctónicas provienen de un crucero de investigación desarrollado en la zona nerítico-costera del centro-sur de Chile a bordo del B/O Kay-Kay, durante los días 10, 11 y 12 de septiembre de 1991. El diseño de muestreo consistió en 6 transectas perpendiculares a la costa, con un total de 38 estaciones de muestreo, circunscritas al rango latitudinal descrito, teniendo como límite longitudinal 25 millas náuticas desde la costa (Fig. $1)$. 


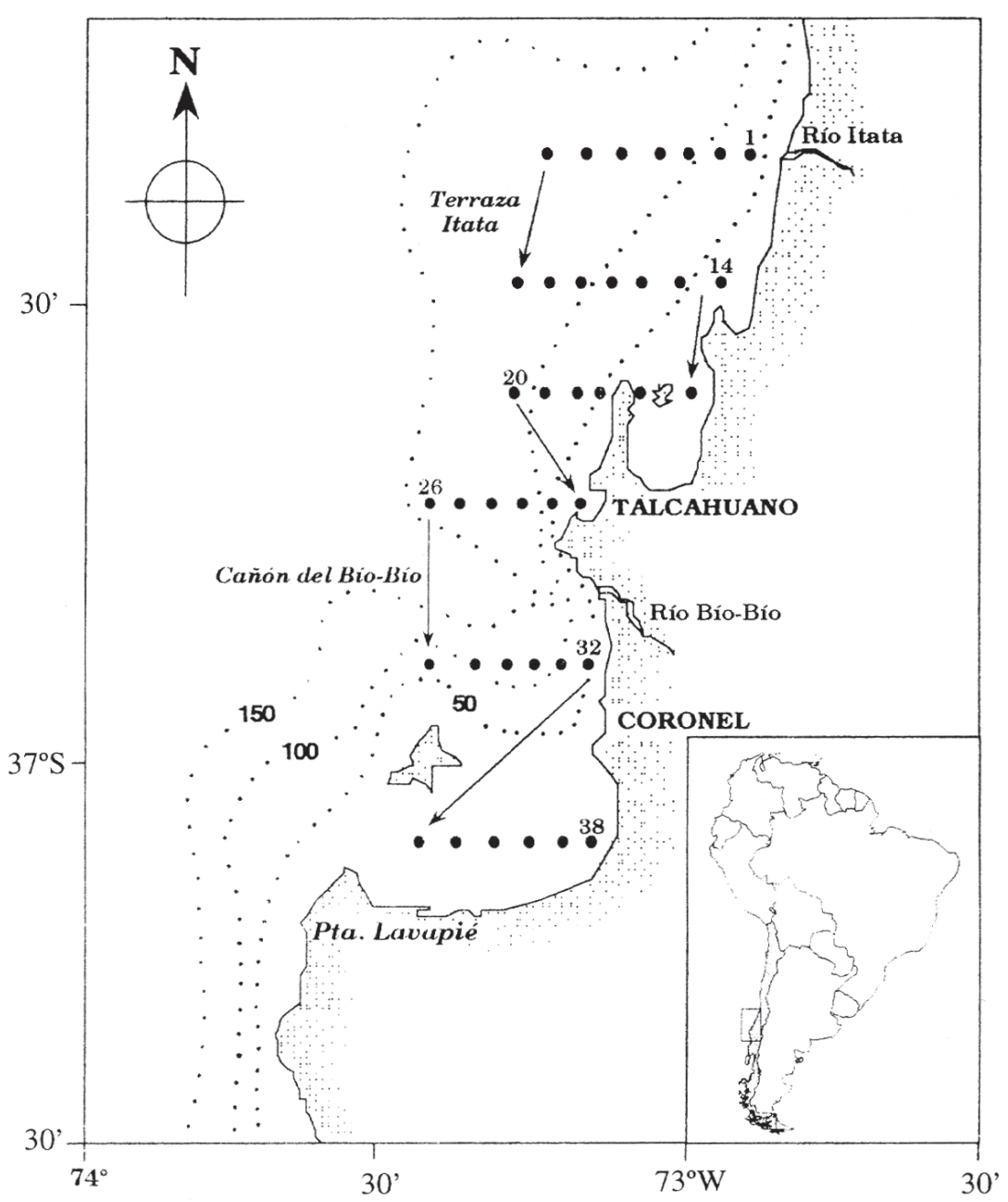

Figura 1. Area de estudio y estaciones de muestreo; se indican además las isobatas de 50,100 y $150 \mathrm{~m}$ de profundidad.

Figure 1. Area of study and sampling stations; isobaths of 50, 100, and $150 \mathrm{~m}$ depth are indicated.

En cada una de las estaciones se realizaron lances oblicuos con una red Bongo de 0,32 m de diámetro de boca, equipada con una malla de $500 \mu \mathrm{m}$ utilizando flujómetros calibrados tipo torpedo General Oceanics. La calibración de los flujómetros se llevó a cabo siguiendo la metodología propuesta por Smith y Richardson (1979). Los lances fueron realizados considerando como profundidad máxima de muestreo la profundidad de ecosonda menos el $10 \%$ (en metros) manteniendo una velocidad del buque que fluctuó entre 1,9 y 2,6 nudos. El ángulo de cable varió entre los $40^{\circ}$ y $50^{\circ}$ y fue medido con un clinómetro manual. El volumen de agua filtrada por ambas redes fluctuó entre 4,70 y 221,13 $\mathrm{m}^{3}$. Aproximadamente el $65 \%$ de los lances fueron nocturnos y durante el desarrollo de cada uno se registró información de carácter básico como número y posición de la estación, fecha y hora, duración del lance, lectura del flujómetro, clinómetro y cable arriado así como también información climática complementaria.

Las muestras planctónicas, obtenidas en cada una de las estaciones, fueron fijadas con formalina al $5 \%$ en agua de mar, neutralizada con bórax y almacenadas en frascos etiquetados de $500 \mathrm{ml}$. 


\section{Análisis de las muestras}

La abundancia de larvas de $M$. gayi gayi fue estandarizada a una unidad de $10 \mathrm{~m}^{2}$ de superficie (Smith y Richardson, 1979), utilizando los datos de abundancia $\left(\mathrm{N}^{\circ} / \mathrm{m}^{3}\right)$, profundidad máxima de cada lance $(\mathrm{m})$ y volumen de agua filtrada por la red $\left(\mathrm{m}^{3}\right)$ (Kramer et al., 1972; Houde, 1977; Smith y Richardson, 1979).

Para lograr una mejor comprensión y definición de la distribución espacial y grado de agrupamiento de los estados larvales, las estaciones fueron ordenadas de acuerdo a Vargas (1995) en tres zonas con notables diferencias en la abundancia promedio: sector norte (transectas 1 y 2), sector centro (transectas 3 y 4) y sector sur (transectas 5 y 6) (Fig. 2).

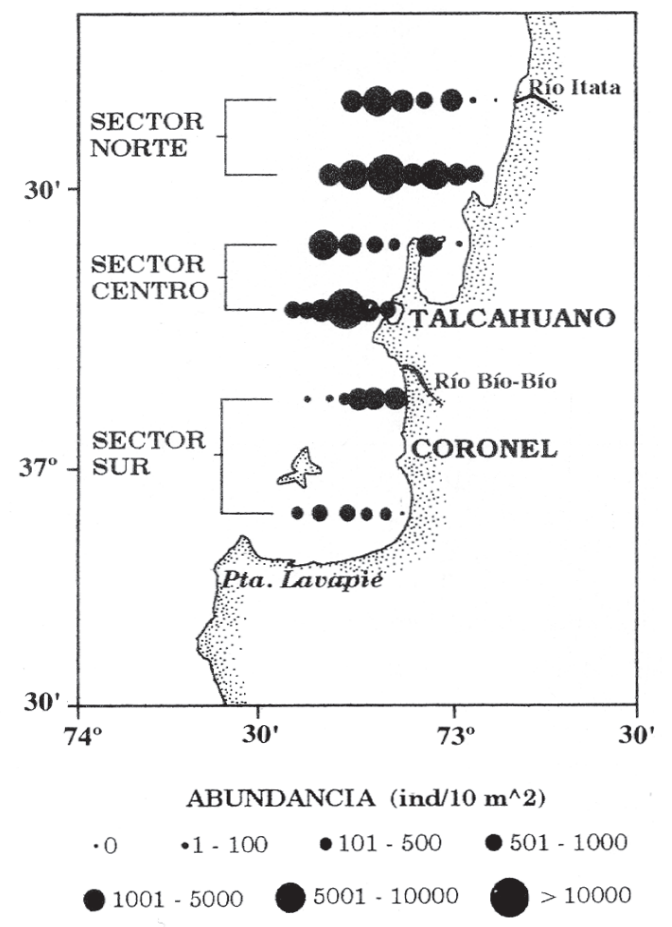

Figura 2. Distribución espacial y abudancia de estados larvales de M. gayi gayi, en tres sectores principales geográficamente delimitados.

Figure 2. Spatial distribution and abundance of larval stages of $M$. gayi gayi, in three main geographically delimited sectors.

\section{Frecuencia de tallas}

La totalidad de las larvas de M. gayi gayi fueron medidas con precisión de $0,1 \mathrm{~mm}$, usando un estereomicroscopio Zeiss (objetivos 50 X). A cada individuo se le registró la longitud estándar (L.E.), medida que comprende desde el borde de la boca hasta el final de la notocorda.

Se realizaron correcciones por encogimiento (formalina) y tiempo de permanencia de ejemplares en la red de muestreo (Theilacker, 1980), así como correcciones por capturas día/noche (o evasión) (Lough et al., 1985; Araya, 1990). Los datos de frecuencia de longitud fueron procesados en términos de tablas de densidad por rango de tallas para cada estación.

\section{Estimación de la mortalidad natural a través del modelo mortalidad-agregación}

Este modelo asume que la depredación como agente causal de mortalidad depende, en un ecosistema acuático, de la distribución espacial tanto de depredadores como de presas (McGurk, 1986). De esta forma, el modelo de interacción mortalidadagregación combina la dependencia del peso descrita por Peterson y Wroblewski (1984), con un índice de agregación espacial de huevos y larvas.

Con el fin de determinar el peso de una larva (peso húmedo) de longitud $\mathrm{x}$, se pesaron en una balanza analítica Sartorius Modelo 1602 MP 8 con precisión 0,0001 g, 200 larvas elegidas al azar, previamente medidas (i.e. LE). El peso seco fue asumido por el modelo como el $20 \%$ del peso húmedo (Peterson y Wroblewski, 1984).

Se realizó un análisis de regresión para obtener la relación longitud-peso y la expresión de dependencia de ambas variables. A partir de la información de densidad de larvas por clase de talla, se procedió a obtener el peso seco larval mediante el uso de la longitud promedio.

El grado de agregación o "patchiness" para cada clase de talla, se cuantificó mediante el uso del índice de Lloyd (Lloyd, 1967), para los tres sectores delimitados con anterioridad (i.e. sectores norte, centro y sur), calculado de acuerdo a la expresión:

$$
\mathrm{p}=1+\left(\sigma^{2} \overline{\mathrm{X}}^{-1}-1\right) \overline{\mathrm{X}}^{-1}
$$


Tabla 1. Regresión simple entre la longitud estándar (log) y el peso seco (log) de larvas de M. gayi gayi.

Table 1. Lineal regresion between dry weight (log) and standard length (log) of M. gayi gayi larvas.

\begin{tabular}{|c|c|c|c|c|}
\hline $\mathrm{n}$ & $\mathrm{R}$ & $\mathrm{R}$ - cuadrado & R-cuadrado ajustado & RMS residual \\
\hline 200 & 0,959 & 0,919 & 0,919 & 0,153 \\
\hline
\end{tabular}

ANÁLISIS DE VARIANZA

\begin{tabular}{|l|c|c|c|c|}
\hline \multicolumn{1}{|c|}{ Fuente } & Grados de libertad & Suma de cuadrados & Cuadrados medios & F - test \\
\hline REGRESION & 1 & 52,323 & 52,323 & 2248,613 \\
\hline RESIDUAL & 198 & 4,607 & 0,023 & $\mathrm{p}=0,0001$ \\
\hline TOTAL & 199 & 56,93 & & \\
\hline
\end{tabular}

donde:

$\overline{\mathrm{X}}=$ media de la densidad de larvas de longitud $x$, $\sigma^{2}=$ varianza de $\bar{X}$.

Este índice mide el actual agrupamiento de un individuo, en relación al agrupamiento promedio esperado en una población dispersa al azar.

Finalmente, la tasa de mortalidad por clase de talla, fue obtenida incorporando los valores de agregación y peso seco en la expresión propuesta por McGurk (1986):

$$
M=-a \times 10^{-3} W^{-b}\left(1+p_{1}\right)
$$

donde:

$\mathrm{M}=$ tasa de mortalidad $\left(\mathrm{d}^{-1}\right)$, predicha por el modelo mortalidad-agregación.

$\mathrm{W}=\operatorname{peso} \operatorname{seco}(\mathrm{g})$ de la clase de talla $x$.

$\mathrm{P}_{1}=$ valor del índice de agregación de Lloyd, para la clase de talla $\mathrm{x}$.

$a=5,26 \mathrm{y}$

$b=0,25$ (constantes empíricas del modelo).

Una vez obtenidos los valores de mortalidad se realizaron análisis estadísticos no paramétricos para determinar la existencia de diferencias significativas en la mortalidad larval a distintas longitudes.

\section{RESULTADOS}

Las larvas tuvieron una longitud estándar que fluctuó entre los 3,3 y 15,5 mm y valores de peso seco $(\mathrm{g})$ entre $2 * 10^{-5}$ y $3 * 10^{-3} \mathrm{~g}$. La relación longitudpeso estuvo descrita por un modelo de tipo potencial, lo que se confirmó al graficar el logaritmo $\left(\log _{10}\right)$ de los datos de peso y longitud (Fig. 3) y obtener una relación lineal con un $r=0,959$ (Tabla $1, \mathrm{p}=0,0001, \mathrm{~F}$-Test). La ecuación así descrita es de la forma:

$$
\mathrm{Y}=4.4055^{-7} * \mathrm{X}^{3,581}
$$

A partir de esta ecuación (Fig. 4) se estimó el peso seco (g) para cada una de las clases descritas en el análisis de frecuencia de tallas.

Debido a la baja abundancia de larvas de $3 \mathrm{~mm}$, la estimación de mortalidad se llevó a cabo abarcando el rango de longitud comprendido entre los 4 y $12 \mathrm{~mm}$ de LE. Con los datos de densidad por rango de longitud y estación de muestreo, se calculó el índice de agregación de Lloyd para cada clase de talla.

La Tabla 2 muestra la densidad promedio, el valor para el índice de agregación de Lloyd, el peso seco y el correspondiente valor de mortalidad natural por clase de talla predicha por este modelo para cada uno de los sectores delimitados.

Se encontraron diferencias significativas en la mortalidad larval en relación a la longitud estándar (Prueba de Kruskal-Wallis; $\mathrm{p}<0,05)$. Los valores de mortalidad fluctuaron entre 0,1 y $0,38 \mathrm{~d}^{-1}$, siendo 


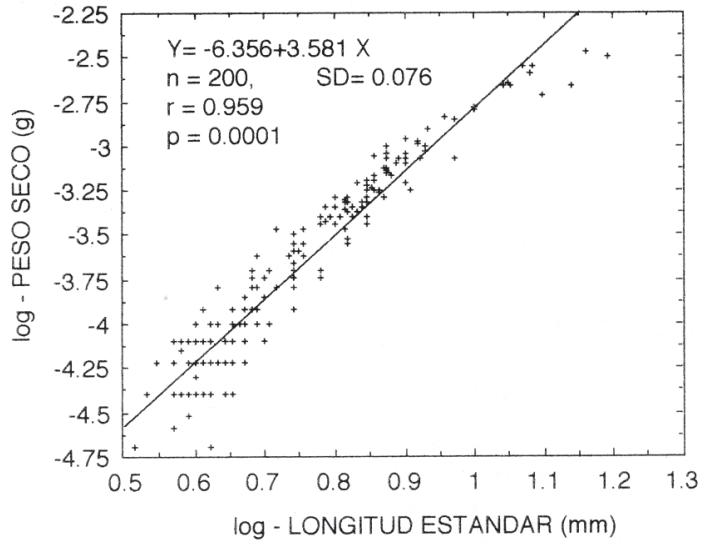

Figura 3. Regresión lineal entre el peso seco y la longitud estándar de larvas de $M$. gayi gayi, previamente transformadas a $\log _{10}$.

Figure 3. Lineal regresion for dry weight and standard lenght of $M$. gayi gayi larvae: data transformed to $\log _{10}$.

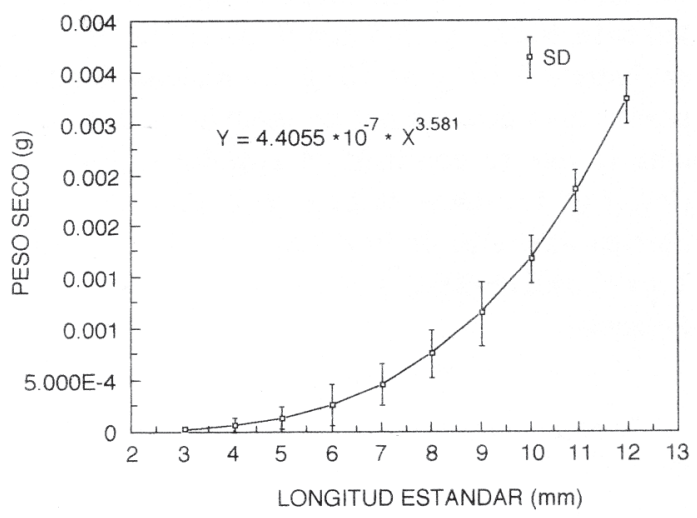

Figura 4. Relación de dependencia entre la longitud estándar y el peso seco de estados larvales de M. gayi gayi.

Figure 4. Dependency relationship between standard length and weight of $M$. gayi gayi larval stages.

mayores en larvas con longitudes entre 4-5 y 9-11 $\mathrm{mm}$ de LE (Fig. 5). Con el objetivo de determinar diferencias estadísticas entre la mortalidad de las larvas a diferentes tallas se realizó la Prueba de Wilcoxon (Sokal y Rohlf, 1969). Esta prueba corrobora lo observado en la Fig. 5, revelando diferencias significativas $(p<0,05)$ en la mortalidad de larvas pequeñas y grandes (4,5 y $11 \mathrm{~mm}$ de LE), res- pecto de las larvas de longitudes intermedias $(6,7 \mathrm{y}$ $8 \mathrm{~mm}$ de LE) (Tabla 3).

La Fig. 6 muestra el grado de agregación larval a diferentes tallas y para cada uno de los sectores geográficos. La relación se ajustó a una curva de tipo polimodal de segundo orden (F-test; $r=0,826$; $\mathrm{p}<0,001$ ), revelando un incremento en la agregación a longitudes mayores a $9 \mathrm{~mm}$ de Le. Para los sectores centro y sur se observa un mayor agrupamiento de larvas con longitudes superiores a $10 \mathrm{~mm}$ de LE, mientras que para el sector norte se hace más evidente el agrupamiento de larvas de 8 y 9 mm de LE.

Para establecer la relación existente entre las variables analizadas (i.e. longitud, agregación y mortalidad), se procedió a ajustar los datos a un gráfico tridimensional de mallas (smoothing technique) con la ayuda del software estadístico STATISTIC IBM Inc. ${ }^{\text {TM }}$. El software grafica los datos reales (i.e. obtenidos en el presente estudio) y los ajusta a una curva que relacione las variables a analizar.

La Fig. 7 muestra para los tres sectores una tendencia similar al relacionar las variables consideradas. El grado de agrupamiento ejerció una gran influencia en los valores de mortalidad natural predichos con este modelo. Para los sectores centro y sur se puede apreciar que a tallas menores $(4-5 \mathrm{~mm}$ de LE) el grado de agrupamiento ejerce una menor influencia en la mortalidad natural, hasta un punto en que ésta alcanza su valor más alto y se mantiene constante independientemente del grado de agregación. A longitudes mayores (> $5 \mathrm{~mm}$ de LE) se puede ver que la agregación ejercería una influencia paulatinamente mayor en la mortalidad, alcanzando niveles casi lineales.

En contraste, para el sector norte y abarcando todo el rango de tallas, se observa un incremento en la mortalidad larval a medida que la agregación aumenta; sin embargo a altos niveles de agregación larval se produce un efecto importante en la mortalidad, la que tiende a disminuir en todo el rango de tallas.

\section{DISCUSION}

Se esperaría que la variabilidad en la alta productividad de las áreas de retención costeras y de latitudes temperadas fuese menor que en el océano abierto (Iles y Sinclair, 1982). Por lo tanto, la falta de ali 
Tabla 2. Densidad promedio (ind/10 $\mathrm{m}^{2}$ ), peso seco (g), agregación espacial y mortalidad natural (M) de M. gayi gayi, por clase de longitud y sectores; se incluyen además otros parámetros estadísticos básicos necesarios para cálculos de mortalidad.

Table 2. Mean density (ind $/ 10 \mathrm{~m}^{2}$ ), dry weight (g), aggregation or spatial patchiness and natural mortality of $M$. gayi gayi, by length class and sector; other basic statistic parameters necessary for mortallity computations are included.

SECTOR NORTE

\begin{tabular}{rrrrrrrr}
\hline $\begin{array}{c}\text { Longitud } \\
(\mathbf{m m})\end{array}$ & $\begin{array}{c}\text { Densidad } \\
\left(\mathrm{ind} / 10 \mathrm{~m}^{2}\right)\end{array}$ & $\mathrm{SD}^{\mathbf{b}}$ & $\mathrm{CV}$ & Agregacion & $\mathrm{n}^{\mathbf{d}}$ & $\begin{array}{c}\mathrm{W}^{\mathbf{e}} \\
(\mathrm{g})\end{array}$ & $\begin{array}{c}\mathrm{M} \\
\left(\mathrm{d}^{-\mathbf{1}}\right)\end{array}$ \\
\hline & & & & & & & \\
4 & 571,796 & 769,707 & 134,615 & 2,810 & 12 & $6,309 * 10^{-5}$ & 0,225 \\
5 & 1874,929 & 2830,334 & 150,957 & 3,278 & 12 & $1,403 * 10^{-4}$ & 0,207 \\
6 & 1278,929 & 1488,950 & 116,422 & 2,355 & 12 & $2,695 * 10^{-4}$ & 0,138 \\
7 & 803,214 & 1188,825 & 148,008 & 3,189 & 13 & $4,681 * 10^{-4}$ & 0,150 \\
8 & 254,714 & 516,578 & 202,807 & 5,109 & 9 & $7,550 * 10^{-4}$ & 0,194 \\
9 & 43,143 & 102,002 & 236,429 & 6,567 & 5 & $1,151 * 10^{-3}$ & 0,216 \\
10 & 67,714 & 90,643 & 133,862 & 2,777 & 4 & $1,679 * 10^{-3}$ & 0,098 \\
11 & 7,0 & 19,776 & 282,509 & 8,838 & 2 & $2,362 * 10^{-3}$ & 0,235 \\
\hline
\end{tabular}

\section{SECTOR CENTRO}

\begin{tabular}{|c|c|c|c|c|c|c|c|}
\hline $\begin{array}{l}\text { Longitud } \\
\text { (mm) }\end{array}$ & $\begin{array}{l}\text { Densidad }^{\mathrm{a}} \\
\left(\mathrm{ind} / 10 \mathrm{~m}^{2}\right)\end{array}$ & $\mathrm{SD}^{\mathrm{b}}$ & $C V^{c}$ & Agregación & $n^{d}$ & $\begin{array}{l}W^{e} \\
(g)\end{array}$ & $\begin{array}{c}\mathrm{M} \\
\left(\mathrm{d}^{-1}\right)\end{array}$ \\
\hline 4 & 2159,833 & 3212,090 & 148,719 & 3,211 & 9 & $6,309 * 10^{-5}$ & 0,249 \\
\hline 5 & 2578,583 & 4197,431 & 162,780 & 3,649 & 10 & $1,403 * 10^{-4}$ & 0,225 \\
\hline 6 & 957,000 & 1016,078 & 106,173 & 2,126 & 8 & $2,695 * 10^{-4}$ & 0,128 \\
\hline 7 & 824,583 & 1252,777 & 151,928 & 3,307 & 8 & $4,681 * 10^{-4}$ & 0,154 \\
\hline 8 & 490,417 & 703,549 & 143,459 & 3,056 & 6 & $7,550 * 10^{-4}$ & 0,129 \\
\hline 9 & 90,500 & 159,960 & 176,751 & 4,113 & 4 & $1,151 * 10^{-3}$ & 0,146 \\
\hline 10 & 56,000 & 131,332 & 234,521 & 6,482 & 2 & $1,679 * 10^{-3}$ & 0,194 \\
\hline 11 & 10,500 & 36,373 & 346,410 & 12,905 & 1 & $2,362 * 10^{-3}$ & 0,332 \\
\hline
\end{tabular}

\section{SECTOR SUR}

\begin{tabular}{crrrrrrr}
\hline $\begin{array}{c}\text { Longitud } \\
(\mathrm{mm})\end{array}$ & $\begin{array}{c}\text { Densidad } \\
\left(\mathrm{ind} / 10 \mathrm{~m}^{2}\right)\end{array}$ & $\mathrm{SD}^{\mathrm{b}}$ & $\mathrm{CV}$ & Agregacion & $\mathrm{n}^{\mathrm{d}}$ & $\begin{array}{c}\mathrm{W}^{\mathrm{e}} \\
(\mathrm{g})\end{array}$ \\
\hline & & & & & & & $\begin{array}{c}\mathrm{M} \\
\left(\mathrm{d}^{-1}\right)\end{array}$ \\
4 & 213,833 & 452,562 & 211,642 & 5,475 & 8 & $6,309 * 10^{-5}$ & 0,382 \\
5 & 481,583 & 826,311 & 171,582 & 3,942 & 10 & $1,403 * 10^{-4}$ & 0,239 \\
6 & 665,250 & 843,923 & 126,858 & 2,608 & 8 & $2,695 * 10^{-4}$ & 0,148 \\
7 & 339,000 & 356,923 & 105,287 & 2,106 & 9 & $4,681 * 10^{-4}$ & 0,111 \\
8 & 110,250 & 122,624 & 111,223 & 2,228 & 7 & $7,550 * 10^{-4}$ & 0,102 \\
9 & 65,333 & 78,469 & 120,105 & 2,427 & 6 & $1,151 * 10^{-3}$ & 0,098 \\
10 & 33,000 & 101,529 & 307,664 & 10,435 & 2 & $1,679 * 10^{-3}$ & 0,297 \\
11 & 21,0 & 58,785 & 279,927 & 8,788 & 2 & $2,362 * 10^{-3}$ & 0,234 \\
\hline
\end{tabular}

$\mathrm{a}$ : Densidad promedio de larvas encontradas en cada sector para una longitud $\mathrm{x}$.

b : Desviación estándar de la muestra.

c : Coeficiente de variación.

d : Número de estaciones positivas para una longitud $\mathrm{x}$.

e : Peso seco de larvas de M. gayi gayi, considerando como el 20\% del peso húmedo (McGurk, 1986). 


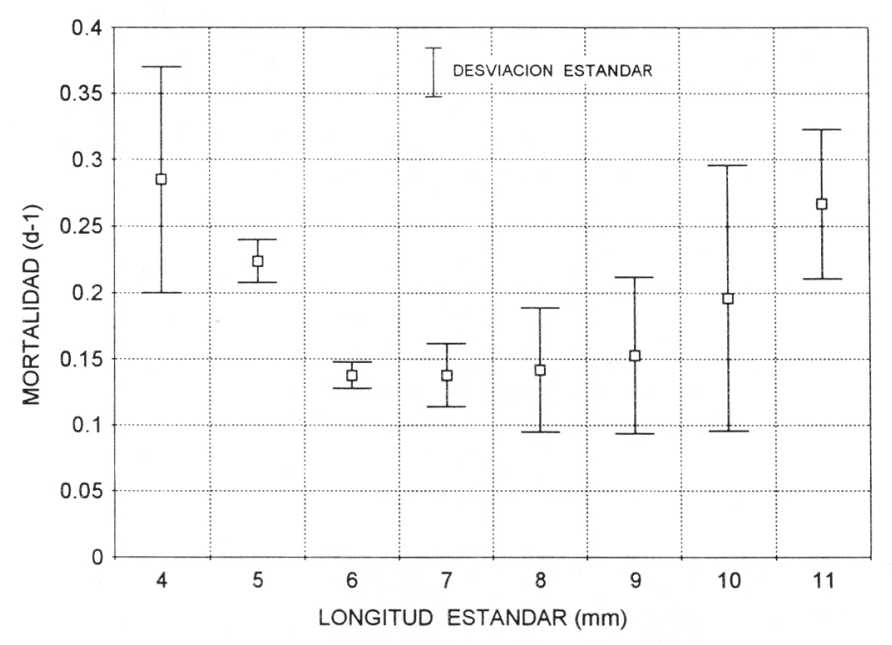

Figura 5. Tasa de mortalidad natural instantánea diaria estimada para diferentes longitudes larvales de $M$. gayi gayi.

Figure 5. Natural instantaneous daily mortality rate estimated for different larval standard lengths of $M$. gayi gayi.

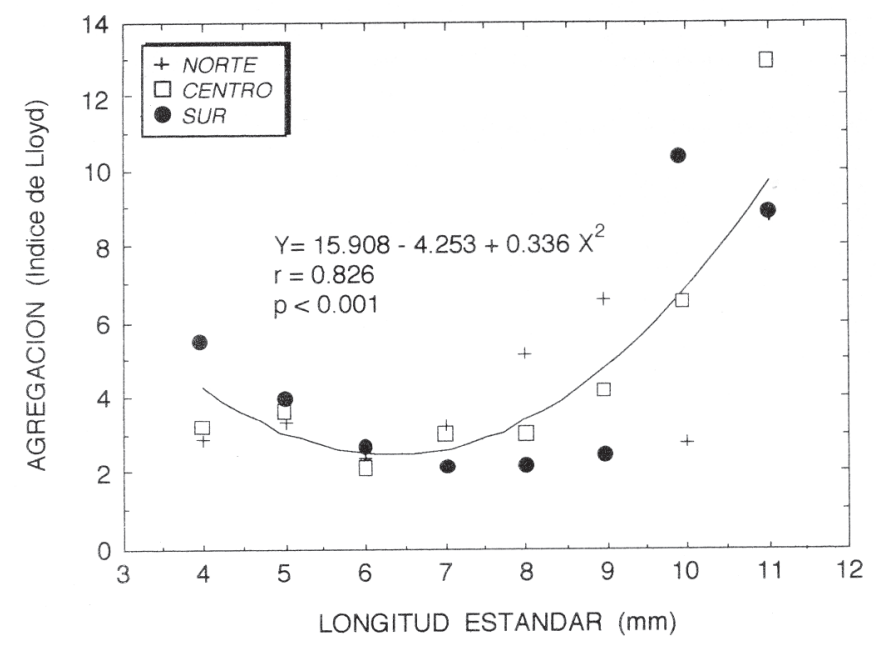

Figura 6. Agregación larval (índice de Lloyd) a diferentes longitudes en tres sectores geográficamente delimitados de $M$. gayi gayi.

Figure 6. M. gayi gayi larval aggregations (Lloyd index) of different lengths, in three geographically delimited sectors.

mento o inanición no sería causal importante de mortalidad larval, a lo menos en las áreas costeras de los sistemas de surgencia, ya que a lo largo del tiempo se garantiza la existencia de parches adecuados de alimento, tras la existencia de períodos de relajación del sistema atmosférico (Sepúlveda, 1990). En este sentido, numerosos investigadores sostienen que la presión de depredación puede lle- gar a ser una fuente de mortalidad mayor que la inanición (e.g., Leak y Houde, 1987). La presión de depredación puede variar debido a cambios en el número total de larvas, preferencia por larvas en relación con otro alimento, o en el grado de concurrencia y agregación de los depredadores en torno a parches de larvas, agregación que sería dependiente de la densidad larval (Rothschield y Rooth, 1982).

Las curvas de agregación-longitud describieron un patrón en forma de "U", es decir, una declinación inicial en los "parches" de larvas recién eclosionadas; seguido de un aumento en la agregación larval a longitudes mayores. Esta tendencia de las larvas más avanzadas a congregarse en cardúmenes (Smith, 1981; McGurk, 1986) se evidencia al observar los resultados del índice de agregación de Lloyd.

Los resultados de la agregación larval corroborarían lo encontrado por Vargas (1995) para el área de estudio, en relación al mayor agrupamiento de larvas de 10-11 mm de LE en los sectores centro y sur, quien sugiere que la presencia de larvas en estados avanzados hacia el saco del Golfo de Arauco, podría deberse a procesos de transporte larval condicionados por la circulación de las aguas sobre la plataforma continental, las que llevan una dirección hacia el sur durante la mayor parte del año (Salinas y Brito, 1993).

La agregación de las larvas en el sector norte del área de estudio estaría influenciando fuertemente a la mortalidad natural, hasta un punto en el cual ésta disminuye a pesar del alto nivel de agregación. La disminución de la mortalidad podría ser un reflejo de las condiciones ambientales que permiten estos altos niveles de agregación. La existencia de parches de larvas en el ambiente, generalmente se encuentra condicionada por procesos oceanográficos como estratificación y frentes, que han sido descritos por Vargas (1995) para el sector norte del área de estudio. La existencia de estos procesos, donde la mezcla entre dos masas de 
Tabla 3. Resultados de la prueba de Wilcoxon $(Z)$ entre las mortalidades de cada una de las ocho clases de talla de $M$. gayi gayi. Se utilizó como réplicas los valores de mortalidad de cada sector $(* \mathbf{p}<0,05)$.

Table 3. Wilcoxon (Z) test for differences between mortalities of each of the eight length classes of M. gayi gayi. The mortality values of each sector were used as replicates $(* p>0,5)$.

Clases de talla $(\mathrm{mm})$

\begin{tabular}{|c|c|c|c|c|c|c|c|}
\hline & 5 & 6 & 7 & 8 & 9 & 10 & 11 \\
\hline 4 & $-1,604 *$ & $-1.604 *$ & $-1,604 *$ & $-1,604 *$ & $-1,604 *$ & $-1,604 *$ & 0 \\
\hline 5 & & $-1,604 *$ & $-1,604 *$ & $-1,604 *$ & $-1,609$ & $-0,535$ & $-1,069$ \\
\hline 6 & & & 0 & 0,535 & -0.535 & $-1,609$ & $-1,604 *$ \\
\hline 7 & & & & 0 & 0 & $-0,535$ & $-1,604^{*}$ \\
\hline 8 & & & & & $-1,069$ & $-0,535$ & $-1,604^{*}$ \\
\hline 9 & & & & & & $-0,535$ & $-1,604^{*}$ \\
\hline 10 & & & & & & & $-1,069$ \\
\hline
\end{tabular}

agua es vigorosa y la producción primaria es alta (Mann y Lazier, 1991), podrían generar ambientes ideales para la sobrevivencia y que según Iles y Sinclair (1982) definen las áreas de retención larval.

Para los sectores centro y sur, el grado de agregación en larvas más pequeñas (4 mm de LE) pareciera sí influenciar fuertemente a la mortalidad hasta un nivel de agregación igual a 6, cuando se hace independiente, a diferencia de lo que ocurre a longitudes mayores ( $>9 \mathrm{~mm}$ de LE) donde la relación puede alcanzar niveles casi lineales en todo el espectro de agregación considerado. En este sentido, la mayor mortalidad observada para larvas en un estado de desarrollo más avanzado, se podría explicar por la alta agregación en que éstas se encuentran, siendo en términos de distribución espacial más vulnerables a la depredación (McGurk, 1986). La influencia de la agregación en la mortalidad observada para larvas más pequeñas, podría revelar que a este estado de desarrollo, otros factores ambientales bióticos o abióticos (e. i., temperatura, concentración de alimento) podrían ejercer una mayor influencia en la mortalidad; como por ejemplo el encontrarse en una etapa crítica en que sus reservas de vitelo se hallan agotado y pasen de una alimentación endógena a una exógena, siendo vital en ese momento la ocurrencia de una concentración adecuada de alimento (Hjort, 1914; FIDE Lasker, 1981).

Los valores de mortalidad obtenidos a través de este modelo $\left(0,1\right.$ a $\left.0,38 \mathrm{~d}^{-1}\right)$, no han podido ser comparados debido a la carencia de información sobre mortalidad de larvas de merluza común para el área de Chile centro-sur. Sepúlveda (1990) en el área de surgencia costera de Chile central, encontró que la mortalidad de larvas de Engraulis ringens y Sardinops sagax estimada en el período marzo-mayo de 1985 sería igual a $0,454 \mathrm{~d}^{-1}$ y $0,870 \mathrm{~d}^{-1}$ respectivamente, y durante el período de máxima abundancia (julio-septiembre de 1985) obtuvo un valor de $\mathrm{Z}$ igual a $1,108 \mathrm{~d}^{-1}$ y $0,838 \mathrm{~d}^{-1}$.

Los valores de mortalidad larval de M. gayi gayi, obtenidos en el presente estudio, son relativamente bajos en comparación a otras especies que forman parte del componente ictioplanctónico típico de esta zona. En concordancia con lo anterior, la máxima actividad reproductiva de la merluza durante los meses de septiembre-octubre (Furet y Lépez, 1984; Amín et al., 1989; Alarcón, 1992), permite que sus larvas estén en el plancton durante un período de productividad ascendente, por lo tanto los niveles de inanición no deberían ser significativos. Herrera y Aguilera (1988) estudiando las larvas de peces de la zona costera de Talcahuano encontraron que los niveles de inanición, evaluados mediante técnicas histológicas, eran poco importantes, sugiriendo que en esta zona, dadas sus particulares condiciones ambientales, la depredación puede ser considerada como el factor más relevante en los niveles de mortalidad.

El exponente del peso seco en el modelo de McGurk (1986) puede ser diferente de $-0,25$ para especies distintas a las que trabajaron Peterson y Wroblewski (1984), pero tal como agrega McGurk (1986), esto no debería afectar la esencia de la hipótesis de mortalidad-agregación, considerando que 

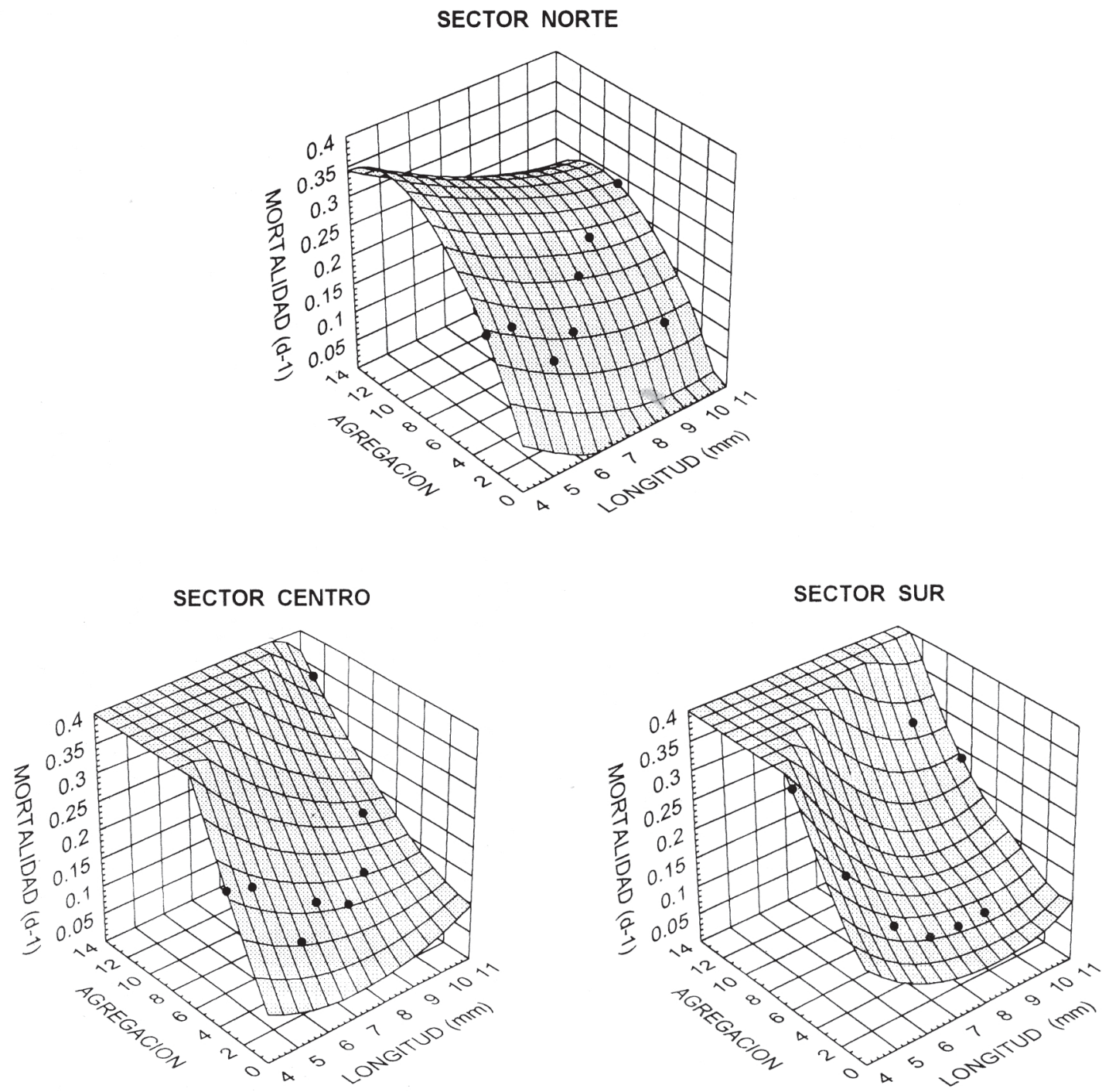

Figura 7. Representación tridimensional de la relación entre la longitud estándar de $M$. gayi gayi, agregación (índice de Lloyd) y mortalidad $\left(\mathbf{d}^{-1}\right)$ larval encontrada en cada uno de los sectores.

Figure 7. Tridimensional representation of the relationship between standard length, aggregation (Lloyd index), and mortality $\left(\mathrm{d}^{-1}\right)$ of $M$. gayi gayi larvae found in each sector.

este autor probó el modelo con información tanto de especies pelágicas como demersales. En este sentido, los valores de mortalidad podrían haberse visto minimizados; sin embargo, la relación agregación-mortalidad existiría como tal.

El modelo de McGurk puede llegar a ser una herramienta que permita comparar valores de mor- talidad en complementación con otros métodos, como por ejemplo el de lectura de anillos de crecimiento. La principal desventaja del modelo de McGurk es el hecho de no considerar la vulnerabilidad-tipo depredador ni el tamaño del depredador (Bailey y Batty, 1983). Sin embargo, su mayor ventaja es el hecho de implicar intrínsecamente la dis- 
minución de la mortalidad por depredación, debido a mezclas turbulentas y mareas, que podrían dispersar tanto huevos como larvas de peces, reduciendo el contacto depredador-presa, pero a la vez dispersando parches de alimento, aumentando por ende las probabilidades de mortalidad por inanición (McGurk, 1986).

\section{AGRADECIMIENTOS}

Los autores expresan sus agradecimientos a la Dirección de Investigación y Desarrollo de la Universidad Austral de Chile (DID-UACH), por el financiamiento del Proyecto S-94-59, "Influencia de los factores ambientales sobre la distribución, abundancia y tasa de crecimiento en larvas de Merluccius gayi gayi, Strangomera bentincki y Engraulis ringens", del cual derivó el presente trabajo.

\section{REFERENCIAS}

Alarcón, R. 1992. Talla de primera madurez sexual y fecundidad parcial en la merluza común, Merluccius gayi gayi, Guichenot, 1848 (Gadiformes - Merluccidae). Informe de Práctica Profesional para optar al título de Biólogo Marino. Universidad de Concepción, 69 pp.

Amín, M., R. Ramón, O. Marín y M. Delpín. 1989. Histología de los ovarios de Merluccius gayi gayi Guichenot, 1848, respecto al desove. Bol. Soc. Biol. Concepción, Chile, 60: 25-34.

Araya, M. 1990. Análisis comparativo de las metodologías de estimación de la tasa instantánea de mortalidad larval en anchoveta (Engraulis ringens, Jenyns) en el norte de Chile. Tesis para optar al título de Biólogo Pesquero. Universidad Arturo Prat, Iquique, Chile, 91 pp.

Arcos, D. 1987. Seasonal and short time-scale variability in copepod abundante and species composition in an upwelling area off Concepción coast, Chile. Ph. D. Dissertation, State University of New York, Stony Brook, 203 pp.

Arcos, D. 1992. Variabilidad, mantención y persistencia de larvas de peces en un ambiente de surgencia costera. In: Actas Resúmenes de las XII Jornadas de Ciencias del Mar, p. 35.
Arcos, D. y N. Navarro. 1986. Análisis de un índice de surgencia para la zona de Talcahuano, Chile (Lat. $37^{\circ} \mathrm{S}$ ). Invest. Pesq. (Chile), 33: 91-98.

Bailey, K.M. y R.S. Batty. 1983. A laboratory study of predation by Aurelia aurita on larval herring (Clupea harengus): Experimental observations compared with model predictions. Mar. Biol. 72: 295-301.

Cáceres, M. y D. Arcos. 1991. Variabilidad en la estructura espacio-temporal de un área de surgencia frente a la costa de Concepción, Chile. Invest. Pesq. (Chile), 36: 27-38.

Furet, L.R. y M.I. Lépez. 1984. Ciclo reproductivo de Merluccius gayi gayi (Guichenot, 1848), mediante estudio histológico de las gónadas. Mem. Asoc. Latinoam. Acuicult., 5 (3): 589-597.

Haury, L.R., J.A. McGowan y P. H. Wiebe. 1978. Pattern and processes in the time-space scales of plankton distributions. In: J. H. Steele (ed.) Spatial pattern in plankton communities. Plenum press, New York and London, pp. 277-327.

Herrera, G. y E. Aguilera. 1988. Larvas de peces de la zona costera de Talcahuano. Composición y variabilidad temporal. In: Actas Resúmenes de las VIII Jornadas de Ciencias del Mar, pp. 36-37.

Hounde, E.D. 1977. Abundante and potencial yield of the Atlantic herring, Opisthotema oglinum, and aspects of its early life history in the eastern Gulf of Mexico. Fish. Bull., U.S., 75 (3): 493-512.

Hounde, E.D. y J.D. Lovdal. 1985. Patterns of variability in ichthyoplankton occurrence and abundance in Biscayne Bay, Florida. Estuar. Coast. Shelf Sci., 20: 79-103.

Hunter, J.R. 1976. Report of a Colloquium on larval fish mortality studies and their relation to fishery research, January 1975. NOAA Tech. Rep. NMFS CIRC-395, 5 pp.

Hunter, J.R. 1981. Feeding ecology and predation of marine fish larvae. In: R. Lasker (ed.) Marine fish larvae: morphology, ecology and relation to fisheries. Wash. Sea Grant Program, Univ. Wash. Press, Seattle, pp. 33-77.

Iles, T.D. y M. Sinclair. 1982. Atlantic herring: stock discreteness and abundance. Science, 215: 627-633. 
Kramer, D., M.J. Kalin, E.G. Stevens, J.R. Thrailkill y J.R. Zweifel. 1972. Collecting and processing data on fish eggs and larvae in the California current region. NOAA Tech. Rep. NMFS CIRC-370, 38 pp.

Lasker, R. 1981. The role of a stable ocean in larval fish survival and subsequent recruitment. In: R. Lasker (ed.) Marine fish larvae: morphology, ecology and relation to fisheries. Wash. Sea Grant Program, University of Washington Press, pp. 8088.

Leak, J.C. y E.D. Houde. 1987. Cohort growth and survival of bay anchovy Anchoa mitchilli larvae in Biscaine Bay, Florida. Mar. Ecol. Progr. Ser., 37: 109-122.

Lloyd, M. 1967. Mean crowding. J. Anim. Ecol., 36: $1-30$.

Lough, R.G., G.R. Bolz, M. Pennington y M.D. Grosslein. 1985. Larval abundance and mortality of Atlantic herring (Clupea harengus L.) spawned in the Georges Bank and Nantucket shoals areas, 1971-1978, in relation to spawning stock size. J. Northw. Atl. Fish. Sci., 6: 21-35.

Mann, K.H. y J.R. Lazier. 1991. Dynamics of marine ecosystems. Biological-physical interactions in the oceans. Blackwell Sci. Publi., Inc., Boston, 486 pp.

McGurk, M.D. 1986. Natural mortality of marine pelagic fish eggs and larvae: role of spatial patchiness. Mar. Ecol. Progr. Ser., 34: 227-242.

Peterson, I. y J.S. Wroblewski. 1984. Mortality rate of fish in the pelagic ecosystem. Can. J. Fish. Aquat. Sci., 41: 1117-1120.

Rothschield, B.J. y C.G.H. Rooth. 1982. Fish ecology III. A foundation for REX. A recruitment experiment. Univ. of Miami Tech. Rep. No. 28, 323 pp.

Salinas, S. y F. Brito. 1993. Corrientes sobre la plataforma continental de Talcahuano. En: Actas Resúmenes de las XIII Jornadas de Ciencias del Mar, p. 39.

Sepúlveda, A. 1990. Variabilidad temporal del ictioplancton en el área de surgencia costera de Chile central: procesos ambientales y biológicos asociados. Tesis para optar al grado de Magíster en Ciencias mención Oceanografía. Universidad de Concepción, 85 pp.

Shepherd, J.G. y D.H. Cushing. 1980. A mechanism for density-dependent survival of larval fish as the basis of a stock-recruitment relationship. J. Cons. Int. Explor. Mer, 39: 160-167.

Smith, P.E. 1973. The mortality and dispersal of sardine eggs and larvae. Rapp. P. -v. Réun. Cons. Int. Explor. Mer, 164: 282-292.

Smith, P.E. y S.L. Richardson. 1979. Técnicas modelo para prospecciones de huevos y larvas de peces pelágicos. FAO, Documentos Técnicos de Pesca No175, FIR/T175 (Es), 107 pp.

Smith, P.E. 1981. Fisheries on coastal pelagic Schooling fish. In: R. Lasker (ed.) Marine Fish Larvae: morphology, ecology and relations to fisheries. Wash. Sea Grant Program. University of Washington Press, pp. 1-31.

Sokal, R.R. y F.J. Rohlf. 1969. Biometry (2nd ed.). W.H. Freeman and Co.; San Francisco, 832 pp.

Theilacker, G.H. 1980. Changes in body measurements of larval northern anchovy, Engraulis mordax, and other fishes due to handling and preservation. Fish. Bull., 78(3): 685-692.

Vargas, C.A. 1995. Influencia de los factores ambientales en la abundancia, distribución espacial y mortalidad de los estados larvales de Merluccius gayi gayi (Guichenot, 1848) en un sistema de surgencia frente a Chile centro-sur. Tesis para optar al título de Biólogo Marino, Univ. Austral de Chile, 82 pp.

Ware, D.M. 1975. Relation between egg size, growth, and natural mortality of larval fish. J. Fish. Res. Bd. Can., 32: 2503-2512.

Wooster, W.S. y J.L. Ried. 1963. Eastern boundary currents. In: M.N. Hill (ed.) The Sea (2), pp. 253 276.

Wroblewski, J.S. 1984. Formulation of growth and mortality larval northern anchovy in a turbulent feeding environment. Mar. Ecol. Prog. Ser., 20: 1322.

Recibido el 26 de marzo de 1996

Aceptado el 8 de noviembre de 1996 